\title{
Diameter dependence of Raman intensities for single-wall carbon nanotubes
}

\author{
L. Alvarez, A. Righi, S. Rols, ${ }^{*}$ E. Anglaret, and J. L. Sauvajol \\ Groupe de Dynamique des Phases Condensées, UMR CNRS 5581, Université Montpellier II, 34095 Montpellier Cedex 5, France \\ E. Muñoz, W. K. Maser, A. M. Benito, and M. T. Martínez \\ Instituto de Carboquímica, CSIC, María de Luna 12, 50015 Zaragoza, Spain \\ G. F. de la Fuente \\ ICMA, CSIC-University of Zaragoza, María de Luna, 50015 Zaragoza, Spain
}

(Received 13 April 2000; revised manuscript received 8 September 2000; published 22 March 2001)

\begin{abstract}
The Raman-active radial breathing modes (RBM) and tangential modes (TM) of single wall carbon nanotubes (SWNT) are studied at fixed laser excitation energy $2.41 \mathrm{eV}(514.5 \mathrm{~nm})$. We focus on the striking diameter dependence of the relative intensity of the TM and RBM between 0.9 and $2.1 \mathrm{~nm}$, which displays a series of plateaux separated by well-defined minima around 1.35 and $0.9 \mathrm{~nm}$. This relates to the diameter dependence of allowed optical transitions (AOT) in SWNT. Diameters in the range 1-1.3 nm (above $1.4 \mathrm{~nm}$ ) correspond to metallic (semiconducting) SWNT in resonance at $2.41 \mathrm{eV}$. The minima correspond to out-ofresonance conditions for TM. The measurement of the diameter dependence of the TM intensity for fixed laser energies is an alternative experimental way to plot the envelopes of the domains of AOT in SWNT.
\end{abstract}

DOI: 10.1103/PhysRevB.63.153401

PACS number(s): 78.30.-j, 61.48.+c

Raman spectroscopy of single wall carbon nanotubes (SWNT) is well known to be a resonant process associated with optical transition between spikes in the one-dimensional (1D) electronic density of states which fall in the visible and near-infrared range. ${ }^{1-6}$ The energy of these allowed optical transitions (AOT) depend both on the diameter and on the metallic or semiconducting character of the tubes, as illustrated in Fig. 1 (from Ref. 7) where the ranges of energies of the AOT have been calculated for semiconducting (black areas) and metallic (dashed areas) tubes. Raman spectroscopy allows us to study the diameter distribution from the analysis of the RBM range ( $\nu$ below $300 \mathrm{~cm}^{-1}$ ) and the electronic properties from the line profiles in the TM range (1400-1700 $\left.\mathrm{cm}^{-1}\right) .{ }^{2-10}$ As far as bundles of SWNT are concerned, intertube coupling must be considered to derive properly the relation between tube diameter and RBM frequency. This was achieved recently by considering a Lennard-Jones potential in addition to a force constant model in order to account for van der Waals intertube interactions. ${ }^{11}$ A significant upshift of the RBM is found for tubes in bundles with respect to isolated tubes [of about $16 \mathrm{~cm}^{-1}$ for a $(10,10)$ SWNT]. The whole calculated data were best fitted by the following nonlinear phenomenologic relation between the RBM frequency and the tube diameter:

$$
\nu_{\mathrm{RBM}}\left(\mathrm{cm}^{-1}\right)=238 / d(\mathrm{~nm})^{0.93} .
$$

Equation (1) has been proposed as a useful tool to estimate tube diameters in SWNT. This was achieved on various samples and a good agreement was evidenced with TEM or neutron diffraction results on the same samples. ${ }^{11,12}$ In Fig. 1 the top scale indicates the RBM frequency calculated from Eq. 1. As far as the TM range $\left(1400-1700 \mathrm{~cm}^{-1}\right)$ is concerned, two distinct profiles corresponding to the specific responses of semiconducting and metallic nanotubes were measured. For semiconducting tubes, the TM essentially dis- plays a symmetric profile with a dominant peak around 1590 $\mathrm{cm}^{-1}$ and two other structures around 1560 and $1550 \mathrm{~cm}^{-1}$. For metallic tubes, the TM displays an intense, broad and asymmetric band around $1540 \mathrm{~cm}^{-1}$, a line at $1560 \mathrm{~cm}^{-1}$, and a sharp peak around $1580 \mathrm{~cm}^{-1} \cdot{ }^{2,4-6,8,9}$ On the other hand, the TM profile was used as a probe to study photoselectively semiconducting or metallic tubes of selected diameter and a good agreement was found with calculations of Fig. 1. ${ }^{5,6}$ Because resonance in Raman can occur via incident or scattered photons, Stokes and anti-Stokes TM spectra can display very distinct profiles when $\left(\nu_{\text {laser }}+\nu_{\mathrm{TM}}\right)$ and $\left(\nu_{\text {laser }}-\nu_{\mathrm{TM}}\right)$ correspond to AOT for tubes of different elec-

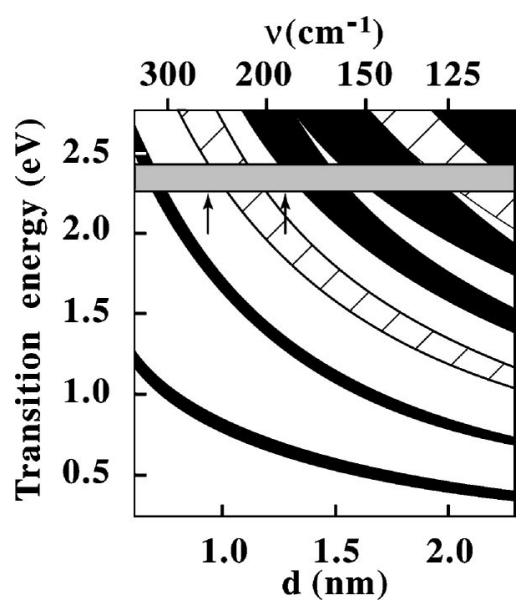

FIG. 1. Allowed optical transitions for SWNT of various diameters and helicities (from Ref. 7). Black and dashed areas correspond to semiconducting and metallic tubes, respectively. The grey frame corresponds to the $2.41 \mathrm{eV}(514.5 \mathrm{~nm})$ laser excitation used in this work: resonance is expected for RBM and TM at the top and bottom of the frame, respectively. The arrows indicate the diameters corresponding to expected minima in the TM intensity (see text). 


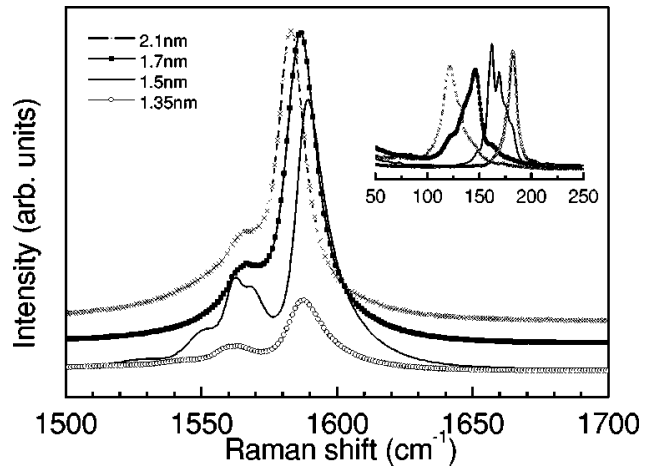

FIG. 2. Raman line shape in the TM range and RBM range (inset) for four samples essentially featured by a single peak in the RBM range. The spectra have been shifted over the vertical scale for clarity.

tronic properties (semiconducting or metallic). ${ }^{7}$ Similarly, resonances in the RBM and TM ranges are expected to be shifted in energy one from each other (Fig. 1). ${ }^{3}$ In this paper we report a micro-Raman investigation performed on selected SWNT samples at fixed laser energy $(2.41 \mathrm{eV})$. We especially focus on samples, or microareas of the samples, featured by a single dominant peak in the RBM range, i.e., a single dominant resonant SWNT, in order to probe the diameter dependence of Raman intensities. The relative intensity of TM and RBM is found to display two plateaux limited by well-defined minima around 1.35 and $0.9 \mathrm{~nm}$. The plateaux correspond to the AOT domains at $2.41 \mathrm{eV}$ (Fig. 1) and the minima correspond to the edges of these domains.

Nanotube samples were prepared in an Ar atmosphere via a $\mathrm{cw}$ laser ablation process using either $\mathrm{Ni} / \mathrm{Y}$ or $\mathrm{Ni} / \mathrm{Co}$ as catalysts ${ }^{13}$ and via the solar route using either $\mathrm{Y}_{2} \mathrm{O}_{3}, \mathrm{La}_{2} \mathrm{O}_{3}$ or $\mathrm{Ni} / \mathrm{Co}$ as catalysts as detailed in Ref. 14. Roomtemperature micro-Raman spectra were measured using the $514.5 \mathrm{~nm}$ excitation line from an Ar ion laser (Spectra physics 2000) in a back scattering geometry on a Jobin-Yvon T64000 spectrometer equipped with a liquid nitrogen cooled CCD detector.

Typical SWNT diameters for samples prepared with the electric arc technique ${ }^{15}$ or pulsed laser ablation technique ${ }^{16}$ are in the range $1.2-1.7 \mathrm{~nm}$. The use of original catalyst mixtures allows one to prepare peculiar samples with different diameter distributions (from 0.9 to $2.1 \mathrm{~nm}$ ) via the solar route. ${ }^{6,14}$ In this range of diameters, the relevant AOT for resonant Raman conditions are the first AOT of metallic tubes and the third and fourth AOT of semiconducting tubes (Fig. 1). The TM range of the Raman spectra measured on four different microareas of the samples is displayed in Fig. 2 . The profile is the usual one observed for semiconducting SWNT with a main peak around $1592 \mathrm{~cm}^{-1}$ and additional bands around $1560 \mathrm{~cm}^{-1}{ }^{1,2}$ The RBM profiles (inset) measured on the same areas are dominated by a single peak, indicating that resonance occurs over a narrow range of diameters. The corresponding diameters calculated from equation 1 are about $2.1 \mathrm{~nm}\left(120 \mathrm{~cm}^{-1}\right), 1.7 \mathrm{~nm}\left(145 \mathrm{~cm}^{-1}\right), 1.5$ $\mathrm{nm}\left(165 \mathrm{~cm}^{-1}\right)$, and $1.35 \mathrm{~nm}\left(180 \mathrm{~cm}^{-1}\right)$, respectively. The main TM peak is observed to harden for diameters decreasing from 2.1 to $1.5 \mathrm{~nm}$. By contrast, for the sample featured

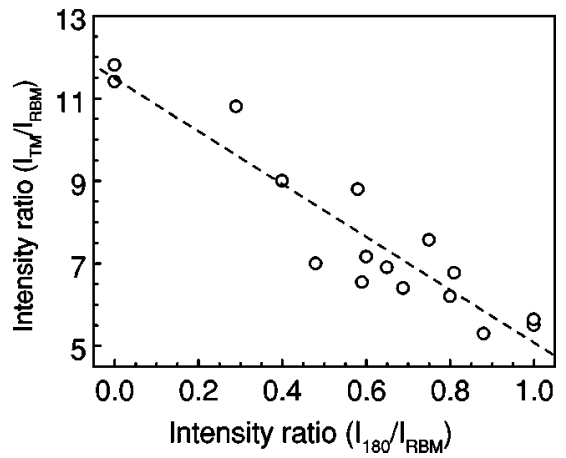

FIG. 3. Dependence of the relative intensities of TM and RBM as a function of the relative intensity of the peak at $180 \mathrm{~cm}^{-1}$ in the RBM bunch.

by $d=1.35 \mathrm{~nm}$ (single peak located at $180 \mathrm{~cm}^{-1}$ ), the frequency is smaller than that expected from the monotonous dependence observed on the other samples (Fig. 2). More striking is the change of the Raman intensities for this sample. For spectra reported in Fig. 2, the relative intensity of the TM and RBM peaks is almost similar for all samples except for $d=1.35 \mathrm{~nm}$ where it is significantly reduced. This is evidence that the resonance behavior at $2.41 \mathrm{eV}$ of SWNT of diameter $d=1.35 \mathrm{~nm}$ is different from that of larger tubes.

A systematic study on a large number of samples has shown that for microareas featured by a single peak below $170 \mathrm{~cm}^{-1}(d>1.35 \mathrm{~nm})$ the integrated TM intensity normalized to the integrated RBM intensity, hereafter called $R_{\text {on }}$, was a constant of about 12. By contrast, for samples featured by a single peak at $180 \mathrm{~cm}^{-1}(d=1.35 \mathrm{~nm})$, this ratio, hereafter called $R_{\text {off }}$, was a constant of about 5. Most of the samples prepared in our groups by the solar route and cw laser ablation are actually characterized by a distribution of RBM between 160 and $180 \mathrm{~cm}^{-1}$. This is also generally observed for samples prepared by electric $\operatorname{arc}^{11,15}$ or pulsed laser ablation. ${ }^{2}$ For all these samples, the value of the TM intensity normalized to the RBM intensity is found to be intermediate between $R_{\text {on }}$ and $R_{\text {off }}$, as illustrated in Fig. 3 where a monotonous dependence of this ratio as a function of the relative intensity of the peak at $180 \mathrm{~cm}^{-1}$ in the RBM bunch is found (Fig. 3). We checked that such a dependence can only be observed with respect to the peak at $180 \mathrm{~cm}^{-1}$. This can be explained in a simple way. Indeed the TM intensity is a mixing of TM from tubes featured by a single peak at $180 \mathrm{~cm}^{-1}$ and tubes featured by RBM peaks below $170 \mathrm{~cm}^{-1}$. Consequently, the TM intensity is written as $I_{\mathrm{TM}}=R_{\mathrm{on}} I_{<170}+R_{\mathrm{off}} I_{180}$, where $I_{180}\left(I_{<170}\right)$ is the integrated RBM intensity of tubes featured by a single peak at 180 $\mathrm{cm}^{-1}$ (peaks below $170 \mathrm{~cm}^{-1}$ ). This implies a linear dependence of the ratio TM intensity/RBM intensity versus $I_{180} / I_{\mathrm{RBM}}$ :

$$
I_{\mathrm{TM}} / I_{\mathrm{RBM}}=R_{\mathrm{on}}-\left(R_{\mathrm{on}}-R_{\mathrm{off}}\right)\left(I_{180} / I_{\mathrm{RBM}}\right) .
$$

Our simple analysis is supported by the dependence observed in Fig. 3. Results in Fig. 3 confirm that the resonant behavior for $d=1.35 \mathrm{~nm}$ is peculiar at $2.41 \mathrm{eV}$ and different 


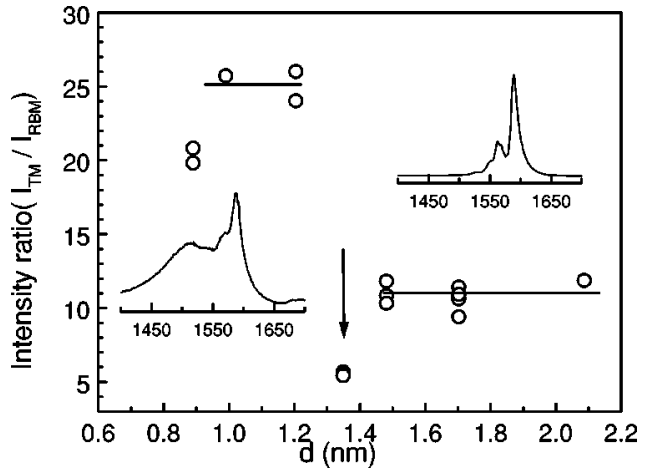

FIG. 4. Diameter dependence of TM intensity normalized to RBM intensity. The arrow indicates the minimum observed for a diameter of $1.35 \mathrm{~nm}$. Insets: typical TM profile for small metallic tubes (left) and large semiconducting tubes (right).

from that of larger tubes. The diameter dependence of the relative intensity can be derived from Raman data obtained on samples featured by a dominant single peak in the RBM range (Fig. 4). For diameters between 1.45 and $2.1 \mathrm{~nm}$ the relative intensity displays a plateau and for $d=1.35 \mathrm{~nm}$ a minimum is observed. All over this diameter range, the profile of the TM is found to be that of semiconducting SWNT, as expected from Fig. 1. The measurements were extended to tubes of small diameters ( $\nu$ above $200 \mathrm{~cm}^{-1}$ ). From Fig. 1, the signature of metallic tubes is expected to appear in the Raman spectrum of these samples because $2.41 \mathrm{eV}$ corresponds to the first AOT of small metallic tubes. This is confirmed by the broad and asymmetric profile of the TM bunch $^{5,6}$ (inset in Fig. 4, left). However, for most of the samples investigated, the peaks above $200 \mathrm{~cm}^{-1}$ were never observed alone but together with peaks around $180 \mathrm{~cm}^{-1}$ which are assigned to semiconducting tubes of diameter $(d$ $=1.35 \mathrm{~nm}$ ). Consequently, in order to estimate the intrinsic contribution from metallic tubes to the TM intensity, we substracted the contribution of semiconducting tubes, i.e., five times the intensity of the peak at $180 \mathrm{~cm}^{-1}$. The ratio between TM and RBM intensities for metallic tubes is of about 25 . It decreases significantly for $d=0.9 \mathrm{~nm}$, indicating that the resonant behavior is also peculiar for this diameter at $2.41 \mathrm{eV}$ (Fig. 4).

The diameter dependence of the TM intensity relates to the peculiar resonance process of the Raman spectrum of SWNT. ${ }^{3,5,7}$ The resonance can occur via incident or scattered photons, the maximum intensity for RBM is shifted of about $0.17 \mathrm{eV}$ from that for TM (Ref. 3) and the high and low limits of the framed domain in Fig. 1 correspond to a maximum of Raman intensity in a Raman Stokes process for RBM and TM, respectively. When both the RBM and TM are in resonance, the relative intensity will be diameter independent. By contrast, for diameters around $1.35 \mathrm{~nm}(0.9 \mathrm{~nm})$, the TM is out-of-resonance for semiconducting (metallic) SWNT while the RBM is still in resonance. This leads to a decrease of the TM intensity. Arrows in Fig. 1 indicate the ranges of diameters where the minima of TM intensity are expected. Consequently, the TM intensity should not be considered as a probe of the sample quality in the characterization of SWNT. Furthermore, the peak position of the TM is not simply dependent on the SWNT diameters. The down shift of the TM for $d=1.35 \mathrm{~nm}$ with respect to the diameter dependence observed for the other samples is actually another consequence of these particular resonance conditions. We assign it to the response of a small amount of larger tubes that are themselves in resonance and contribute to lower frequencies (see spectra in Fig. 2). The good agreement between experimental results and calculations from Fig. 1 allows one to state the validity of the latter. Previous calculations of the diameter and helicity dependence of the AOT were reported by Kataura et al. ${ }^{5}$ using a value of the overlap integral of $2.7 \mathrm{eV}$. They found that the low limit of the AOT domain for semiconducting tubes at $2.41 \mathrm{eV}$ corresponds to a diameter of $1.2 \mathrm{~nm}$ (RBM around $200 \mathrm{~cm}^{-1}$ ), in disagreement with our experimental results. The value 2.9 $\mathrm{eV}$ for the overlap integral ${ }^{7}$ leads to a better agreement with experimental data.

In summary, a striking diameter dependence of the Raman intensities at fixed laser energy has been demonstrated. The relative intensity of TM and RBM displays a series of plateaux separated by well-defined minima. This is explained by the peculiar Raman resonant conditions for semiconducting and metallic SWNT. A comparable "oscillatory"' behavior of the energy dependence of the RBM profile at fixed SWNT diameters was recently evidenced. ${ }^{17}$ Reciprocally, the measurement of the diameter dependence of the TM intensity at fixed laser energies provides an alternative experimental tool to plot the envelopes of the domains of AOT in SWNT.

We thank T. Guillard and D. Laplaze for their help in the sample synthesis. A.R. acknowledges financial support from the Brazilian agency CNPq. S.R. acknowledges the Région Languedoc-Roussillon for financial support. E.M. acknowledges financial support from the Departamento de Educación y Cultura de la Comunidad Autónoma de Aragón.
*Also at Institut Laue-Langevin, 38042 Grenoble, France

${ }^{1}$ R. Saito, G. Dresselhaus, and M. S. Dresselhaus, Physical Properties of Carbon Nanotubes (Imperial College Press, London, 1998); M. S. Dresselhaus and P. C. Eklund, Adv. Phys. 49, 705 (2000).

${ }^{2}$ A. M. Rao, E. Richter, S. Bandow, B. Chase, P. C. Eklund, K. A. Williams, S. Fang, K. R. Subbaswamy, M. Menon, A. Thess, R. E. Smalley, G. Dresselhaus, and M. S. Dresselhaus, Science 275, 187 (1997).
${ }^{3}$ E. Richter and K. R. Subbaswany, Phys. Rev. Lett. 79, 2738 (1997).

${ }^{4}$ M. A. Pimenta, A. Marucci, S. D. M. Brown, M. J. Matthews, A. M. Rao, P. C. Eklund, R. E. Smalley, G. Dresselhaus, and M. S. Dresselhaus, J. Mater. Res. 13, 2396 (1998); M. A. Pimenta, A. Marucci, S. A. Empedocles, M. G. Bawendi, E. B. Hanlon, A. M. Rao, P. C. Eklund, R. E. Smalley, G. Dresselhaus, and M. S. Dresselhaus, Phys. Rev. B 58, R16 016 (1998).

${ }^{5}$ H. Kataura, Y. Kumazawa, Y. Maniwa, I. Umezu, S. Suzuki, Y. 
Ohtsuka, and Y. Achiba, Synth. Met. 103, 2555 (1999).

${ }^{6}$ L. Alvarez, A. Righi, T. Guillard, S. Rols, E. Anglaret, D. Laplaze, and J.-L. Sauvajol, Chem. Phys. Lett. 316, 186 (2000).

${ }^{7}$ S. D. M. Brown, P. Corrio, A. Marucci, M. S. Dresselhaus, M. A. Pimenta, and K. Kneipp, Phys. Rev. B 61, R5137 (2000).

${ }^{8}$ M. Sugano, A. Kasuya, K. Tohji, Y. Saito, and Y. Nishina, Chem. Phys. Lett. 292, 575 (1998).

${ }^{9}$ A. Kasuya, Y. Sasaki, Y. Saito, K. Tohji, and Y. Nishina, Phys. Rev. Lett. 78, 4434 (1997); A. Kasuya, M. Sugano, T. Maeda, Y. Saito, K. Tohji, H. Takahashi, Y. Sasaki, M. Fukushima, Y. Nishina, and C. Horie, Phys. Rev. B 57, 4999 (1997).

${ }^{10}$ E. Anglaret, N. Bendiab, T. Guillard, C. Journet, G. Flamant, D. Laplaze, P. Bernier, and J. L. Sauvajol, Carbon 36, 1815 (1998).

${ }^{11}$ S. Rols, A. Righi, L. Alvarez, E. Anglaret, R. Almairac, C. Journet, P. Bernier, J. L. Sauvajol, A. M. Benito, W. K. Maser, E. Muñoz, M. T. Martinez, G. F. de la Fuente, A. Girard, and J. C. Ameline, Eur. Phys. J. B 18, 201 (2000).

${ }^{12}$ S. Rols, R. Almairac, L. Henrard, E. Anglaret, and J. L. Sauvajol,
Eur. Phys. J. B 10, 263 (1999).

${ }^{13}$ W. K. Maser, E. Muñoz, A. M. Benito, M. T. Martinez, G. F. de la Fuente, Y. Maniette, E. Anglaret, and J. L. Sauvajol, Chem. Phys. Lett. 292, 587 (1998); E. Muñoz, W. K. Maser, A. M. Benito, M. T. Martinez, G. F. de la Fuente, Y. Maniette, A. Righi, E. Anglaret, and J. L. Sauvajol, Appl. Phys. A: Mater. Sci. Process. 70, 145 (2000).

${ }^{14}$ L. Alvarez, T. Guillard, G. Flamant, D. Laplaze, and J. L. Sauvajol, Appl. Phys. A: Mater. Sci. Process. 70, 169 (2000).

${ }^{15}$ C. Journet, W. K. Maser, P. Bernier, A. Loiseau, M. Lamy de la Chapellee, S. Lefrant, P. Deniard, R. Lee, and J. E. Fischer, Nature (London) 388, 756 (1997).

${ }^{16}$ A. Thess, R. Lee, P. Nikolaev, H. Dai, P. Petit, J. Robert, C. Xu, Y. H. Lee, S. G. Kim, A. G. Rinzler, D. T. Colbert, G. E. Scuseria, D. Tomanek, J. E. Fischer, and R. E. Smalley, Science 273, 483 (1996).

${ }^{17}$ M. Milnera, J. Kurti, M. Hulman, and H. Kuzmany, Phys. Rev. Lett. 84, 1324 (2000). 